МОЛЕКУЛЯРНО-ДИНАМИЧЕСКОЕ

\title{
МОДЕЛИРОВАНИЕ ЛОКАЛЬНОЙ
}

СТРУКТУРЫ РАСПЛАВОВ Аl-Cu В ЖИДКОМ И ПЕРЕОХЛАЖДЕННОМ СОстояниях

\author{
${ }^{1,2}$ МЕНЬШИКОВА С. Г. \\ ${ }^{1}$ Удмуртский федеральный исследовательский центр Уральского отделения РАН, \\ 426067, г. Ижевск, ул. Т. Барамзиной, 34 \\ ${ }^{2}$ Удмуртский государственный университет, 426034, г. Ижевск, ул. Университетская, 1
}

\begin{abstract}
АННОТАЦИЯ. Методом классической молекулярной динамики с помощью потенциала погруженного атома рассчитаны атомные траектории для сплавов $\mathrm{Al}-\mathrm{Cu}$ в диапазоне составов от 0 до 28 ат.\% Cu. Конфигурации рассчитаны при изотермической выдержке для различных температур как в области равновесного расплава, так и для переохлажденной жидкости. Проведен анализ термодинамических и структурных свойств системы. Рассчитаны значения плотности расплавов. Рост концентрации $\mathrm{Cu}$ в расплаве $\mathrm{Al}$ приводит к нелинейному росту значений плотности.
\end{abstract}

КЛЮЧЕВЫЕ СЛОВА: жидкий сплав, ближний порядок, структура, концентрация, параметр Вендта-Абрахама, параметр трансляционного порядка, переохлажденный расплав.

\section{ВВЕДЕНИЕ}

Сплавы системы $\mathrm{Al}-\mathrm{Cu}$ находят широкое применение в различных областях промышленности вследствие высокой механической прочности при высоких температурах, небольшому весу, высоких износостойкости и коррозионной стойкости. Несмотря на большой объем экспериментальных и теоретических исследований, описанных в литературе, в частности, [1 - 13], особенности сплавов данной системы до сих пор являются предметом многочисленных научных дискуссий, особенно это касается жидкой фазы и процессов затвердевания (кристаллизационной и стеклообразующей способности). Структурное состояние расплава совместно с высокоскоростным охлаждением, как известно, оказывают влияние на морфологию формирующихся структур при затвердевании [14]. Возникает необходимость исследования структурного состояния расплавов в широком диапазоне температур. В большинстве случаев структура и свойства получаемых материалов зависят от типа атомного упорядочения, реализуемого в жидкой фазе, а некоторые теплофизические свойства (теплопроводность, вязкость, электрическое сопротивление, магнитная восприимчивость и т.п.) определяются соотношением концентраций компонентов - атомов $\mathrm{Al}$ и $\mathrm{Cu}[15-18]$. Исследование жидкой фазы открывает возможности управления формированием структур на микроскопических масштабах [19 - 20].

В настоящей работе выполняется анализ термодинамических и структурных свойств системы $\mathrm{Al}-\mathrm{Cu}$ (до 28 ат.\% $\mathrm{Cu}$ ) при различных температурах на основе молекулярнодинамических расчетов. В выбранной области исследований на диаграмме состояний [21] (рис. 1) отмечено наличие эвтектической точки при концентрации $\mathrm{Cu} \sim 17.1$ ат.\%. Таким образом, рассматриваемые расплавы относятся к эвтектическому типу. Известно, что подобные сплавы вследствие их уникальных физико-химических свойств находят широкое применение в различных отраслях производства.

Равновесная диаграмма состояний системы $\mathrm{Al}-\mathrm{Cu}$ в области богатой алюминием, представлена на рис. 1, а температуры выбранных изотермических выдержек-отжигов в исследовании (изотермы) отмечены штриховыми линиями. 


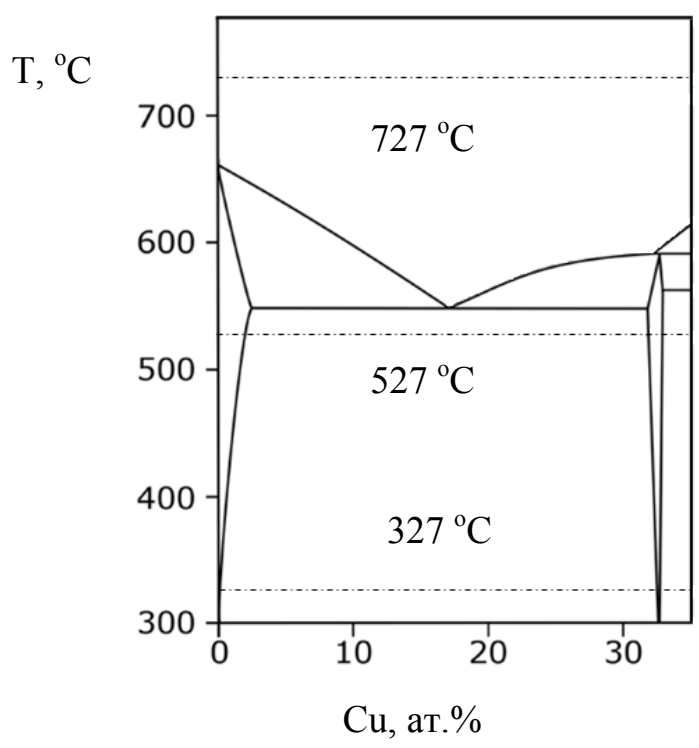

Рис. 1. Диаграмма состояний $\mathrm{Al}-\mathrm{Cu}$, область, богатая алюминием [21].

Прерывистой линией отмечены изотермы, при которых выполнены расчеты

\section{МЕТОДИКА МОДЕЛИРОВАНИЯ}

Молекулярно-динамические расчеты произведены в пакете LAMMPS. Исследуемая система состояла из $27^{3}=19986$ атомов, взаимодействующих посредством ЕАМ потенциала, предложенного в [25]. Поскольку нами выбрана хорошо изученная система Al-Cu, для расчетов мы воспользовались известными литературными данными [25], используя три функции парного взаимодействия (Al-Al, $\mathrm{Al}-\mathrm{Cu}, \mathrm{Cu}-\mathrm{Cu})$, две функции погружения и две функции распределения плотности электронных облаков. В соответствии с данными работы [25], потенциальная энергия $i$-го атома для расплавов $\mathrm{Al}-\mathrm{Cu}$ может быть выражена соотношением

$$
E_{i}=F_{\alpha}\left(\sum_{i \neq j} \rho_{\alpha}\left(r_{i j}\right)\right)+\frac{1}{2} \sum_{i \neq j} \varphi_{\alpha \beta}\left(r_{i j}\right),
$$

где $r_{i j}$ - расстояние между $i$-м и $j$-м атомами, $\varphi_{\alpha \beta}-$ функция парного потенциала, $\rho_{\alpha}-$ вклад в плотность заряда электронов от $j$-го атома в место расположения $i$-го атома и $F$-это функция "погружения", которая представляет энергию, необходимую для помещения $i$-го атома типа $\alpha$ в электронное облако. Индексами " $\alpha$ " и " $\beta$ " обозначены типы элементов, входящих в состав исследуемого металлического сплава, а именно, $\mathrm{Al}$ и $\mathrm{Cu}$. Моделирование выполнено в NpT-ансамбле при нулевом давлении. Рассмотрено 15 различных составов $\mathrm{Al}_{100-\mathrm{x}} \mathrm{Cu}_{\mathrm{x}}(\mathrm{x}=0,2, \ldots, 28$ ат.\%). Для каждого состава расчет проводился в четыре этапа. Первый этап - создание начальной конфигурации в виде кубической решетки со случайным распределением элементов по узлам, минимизация данной конфигурации и последующая релаксация при температуре $\mathrm{T}=1800 \mathrm{~K}\left(1527^{\circ} \mathrm{C}\right)$; второй этап - релаксация системы при температуре $1000 \mathrm{~K}\left(727^{\circ} \mathrm{C}\right)$ и затем рабочий счет со сбором данных и записью в файл траекторий; третий этап-релаксация системы при температуре $800 \mathrm{~K}\left(527^{\circ} \mathrm{C}\right)$ и снова рабочий счет со сбором данных и записью файла траекторий; четвертый этап - релаксация системы при температуре $600 \mathrm{~K}\left(327^{\circ} \mathrm{C}\right)$ и вновь рабочий счет со сбором данных и записью файла траекторий.

Исследование структурных особенностей исследуемых расплавов выполняли с помощью радиальной функции распределения $\boldsymbol{g}(\boldsymbol{r})$ (ФРPА) [22]. В качестве дополнительного параметра, характеризующего структурное упорядочение расплава, использовали параметр Вендта-Абрахама $r^{W A}[23]$ :

$$
r^{W A}=\frac{g_{\min }(r)}{g_{\max }(r)},
$$


где $g_{\max }(r)$ и $g_{\min }(r)$ - значения первого максимума и первого минимума радиальной функции распределения частиц соответственно. Уменьшение значения $r^{W A}$ характеризует увеличение степени локального структурного порядка в системе [23]. Также информацию о структурных особенностях исследуемой системы получали с помощью параметра трансляционного порядка [24]:

$$
T P=\int_{0}^{r_{0}}|g(r)-1| d r,
$$

где $r_{0}$-расстояние, которым ограничивается рассмотрение структурных особенностей системы. Увеличение параметра TP указывает на усиление структурного упорядочения в системе [24].

\section{РЕЗУЛЬТАТЫ МОДЕЛИРОВАНИЯ}

На рис. $2(a-b)$ представлены полные ФРРА исследованных расплавов $\mathrm{Al}-\mathrm{Cu}$ при температурах $1000 \mathrm{~K}(a)$ - равновесный расплав, 800 и $600 \mathrm{~K}(\sigma, 8)$ - переохлажденный расплав. Анализ ФРРА при температурах 1000 и 800 К показал, что локальные структуры расплава одного и того же состава при данных температурах идентичны, справедливо для всех составов. В таблице представлены координационные числа соответствующих пар атомов для равновесных и переохлажденных расплавов. Как видно из таблицы, независимо от температуры, рост концентрации меди от 0 до 28 ат.\% в расплаве приводит к довольно монотонному уменьшению среднего координационного числа в парах (Al-Al) от 12 до 9 и, соответственно, росту в парах $(\mathrm{Al}-\mathrm{Cu})$ и парах $(\mathrm{Cu}-\mathrm{Cu})$ от 0 до 4. Рис. 3 показывает, что наиболее вероятное межатомное расстояние (первый пик полной ФРРА) с увеличением содержания меди в расплаве уменьшается от 2.81 до $2.72 \AA \AA$ немонотонно при переходе через эвтектическую точку. Т.к. сплавы $\mathrm{Al-Cu}$ характеризуются сильным взаимодействием между разноименными компонентами, можно предположить существование преимущественного соседства в расплавах разносортных атомов с ростом содержания меди. При температуре $600 \mathrm{~K}$, как видно из рис. 2, в, переохлажденный расплав при некоторых концентрациях стабилен на временах моделирования, а при некоторых составах, в частности, $\mathrm{Al}_{92} \mathrm{Cu}_{18}$ (состав, близкий к эвтектическому) - кристаллизуется, что может быть связано с зависимостью кристаллизационной стабильности исследованной системы от состава и вызывает интерес к дальнейшему исследованию.

Таблица

Координационное число $Z_{i-j}{ }^{*}$ в расплавах $\mathrm{Al}-\mathrm{Cu}$ при различных температурах

\begin{tabular}{|c|c|c|c|c|c|c|}
\hline \multirow{2}{*}{$\begin{array}{c}\text { Концентрация } \\
\text { Сu, ат.\% }\end{array}$} & \multicolumn{2}{|c|}{$Z(\mathrm{Al}-\mathrm{Al})$} & \multicolumn{2}{c|}{$Z(\mathrm{Al}-\mathrm{Cu})$} & \multicolumn{2}{c|}{$Z(\mathrm{Cu}-\mathrm{Cu})$} \\
\cline { 2 - 7 } & $1000 \mathrm{~K}$ & $800 \mathrm{~K}$ & $1000 \mathrm{~K}$ & $800 \mathrm{~K}$ & $1000 \mathrm{~K}$ & $800 \mathrm{~K}$ \\
\hline 0 & $12.7 \pm 0.2$ & $12.9 \pm 0.2$ & - & - & - & - \\
\hline 2 & $12.3 \pm 0.1$ & $12.8 \pm 0.1$ & $0.2 \pm 0.1$ & $0.3 \pm 0.1$ & $0.1 \pm 0.1$ & $0.1 \pm 0.2$ \\
\hline 4 & $12.0 \pm 0.1$ & $12.6 \pm 0.1$ & $0.5 \pm 0.2$ & $0.5 \pm 0.2$ & $0.3 \pm 0.1$ & $0.3 \pm 0.1$ \\
\hline 6 & $11.9 \pm 0.1$ & $12.4 \pm 0.2$ & $0.7 \pm 0.1$ & $0.8 \pm 0.2$ & $0.4 \pm 0.1$ & $0.4 \pm 0.2$ \\
\hline 8 & $11.8 \pm 0.1$ & $12.0 \pm 0.1$ & $0.9 \pm 0.1$ & $1.0 \pm 0.1$ & $0.6 \pm 0.1$ & $0.5 \pm 0.2$ \\
\hline 10 & $11.7 \pm 0.1$ & $11.7 \pm 0.1$ & $1.3 \pm 0.2$ & $1.3 \pm 0.2$ & $0.7 \pm 0.2$ & $0.7 \pm 0.2$ \\
\hline 12 & $11.5 \pm 0.2$ & $11.6 \pm 0.1$ & $1.6 \pm 0.1$ & $1.6 \pm 0.2$ & $0.9 \pm 0.1$ & $0.8 \pm 0.2$ \\
\hline 14 & $11.3 \pm 0.2$ & $11.3 \pm 0.2$ & $1.8 \pm 0.1$ & $1.9 \pm 0.1$ & $1.1 \pm 0.2$ & $1.1 \pm 0.2$ \\
\hline 16 & $11.0 \pm 0.2$ & $11.1 \pm 0.1$ & $2.1 \pm 0.1$ & $2.1 \pm 0.2$ & $1.2 \pm 0.1$ & $1.2 \pm 0.1$ \\
\hline 18 & $10.6 \pm 0.1$ & $10.7 \pm 0.2$ & $2.2 \pm 0.2$ & $2.4 \pm 0.2$ & $1.4 \pm 0.2$ & $1.4 \pm 0.2$ \\
\hline 20 & $10.3 \pm 0.1$ & $10.6 \pm 0.1$ & $2.6 \pm 0.1$ & $2.7 \pm 0.2$ & $1.6 \pm 0.2$ & $1.6 \pm 0.2$ \\
\hline 22 & $10.2 \pm 0.1$ & $10.3 \pm 0.2$ & $2.9 \pm 0.1$ & $2.9 \pm 0.1$ & $1.9 \pm 0.2$ & $1.8 \pm 0.1$ \\
\hline 24 & $9.9 \pm 0.1$ & $10.1 \pm 0.1$ & $3.2 \pm 0.1$ & $3.2 \pm 0.2$ & $2.1 \pm 0.2$ & $2.1 \pm 0.1$ \\
\hline 26 & $9.6 \pm 0.1$ & $9.7 \pm 0.1$ & $3.6 \pm 0.1$ & $3.6 \pm 0.2$ & $2.3 \pm 0.2$ & $2.3 \pm 0.2$ \\
\hline 28 & $9.5 \pm 0.1$ & $9.4 \pm 0.2$ & $3.9 \pm 0.1$ & $3.9 \pm 0.1$ & $2.6 \pm 0.2$ & $2.6 \pm 0.1$ \\
\hline * получены из кумулятивных ФРРА соответствующих пар атомов.
\end{tabular}



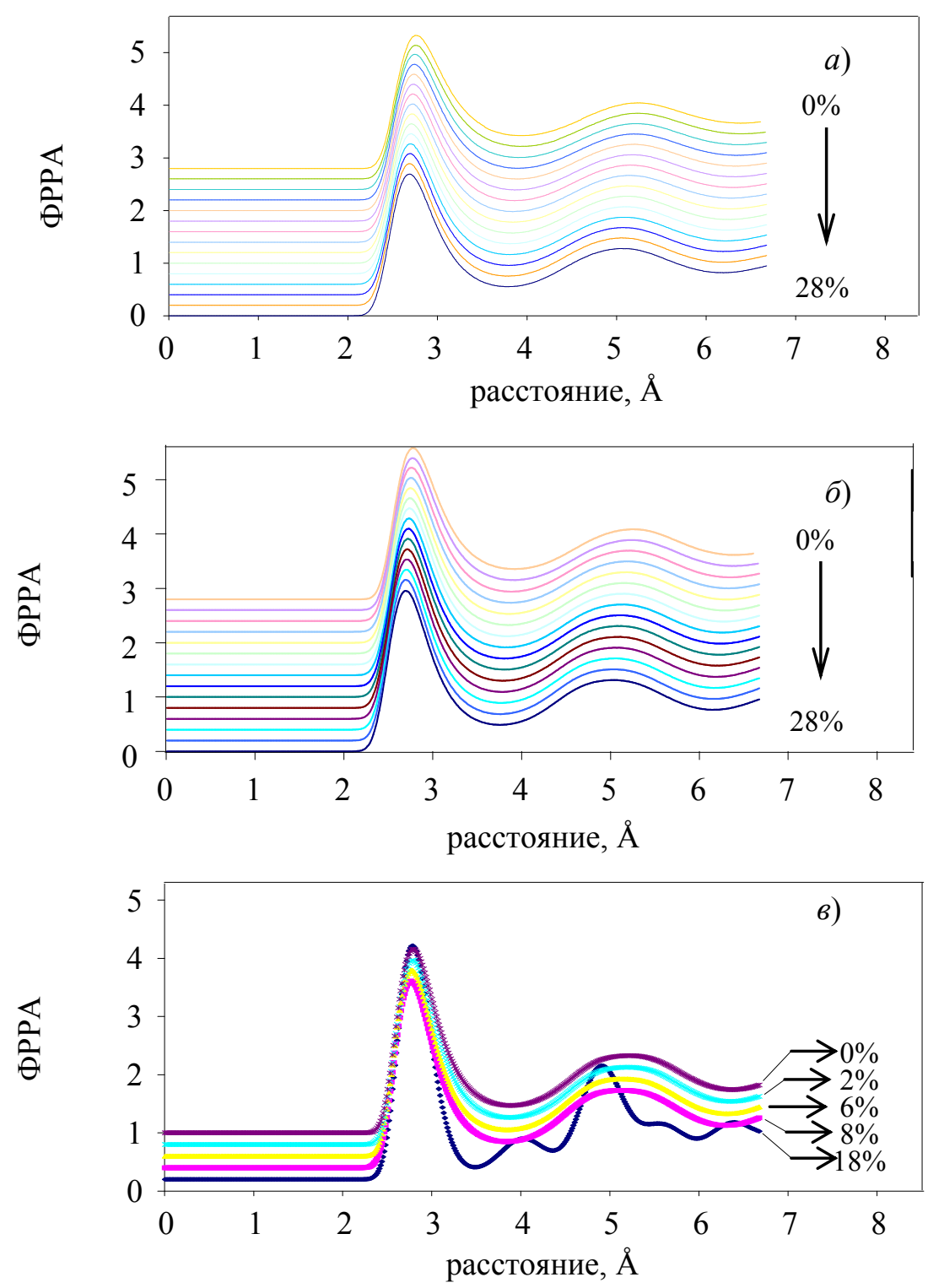

Рис. 2. ФРРА исследованных расплавов Аl-Сu при температурах: 1000 К (a) - равновесный расплав; 800 К (б) и 600 К (в) - переохлажденный расплав. Указана концентрация меди в расплаве (ат.\%)

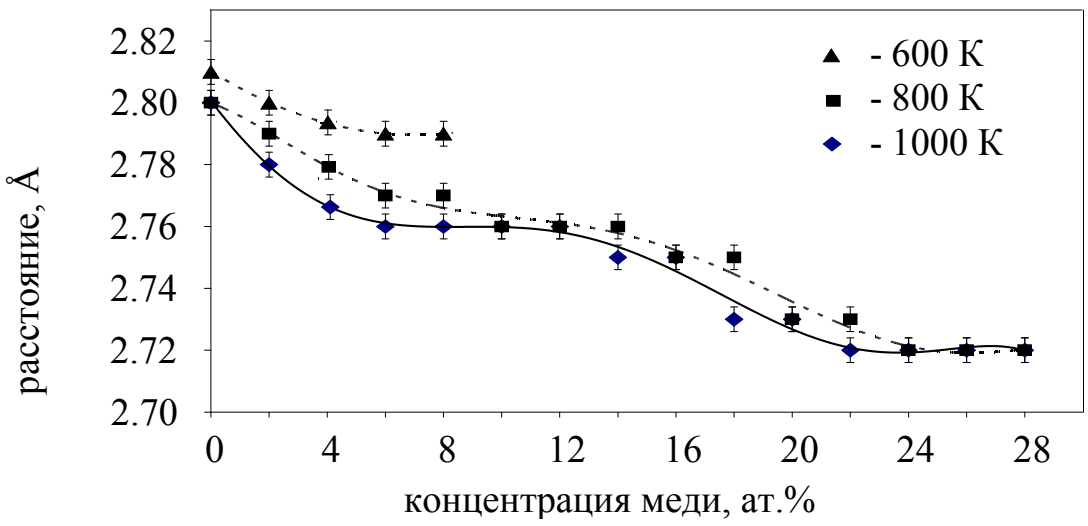

Рис. 3. Наиболее вероятное межатомное расстояние в зависимости от концентрации меди в расплаве (аппроксимация полиномом 6-й степени) 
На рис. 4 представлены концентрационные зависимости параметра Вендта-Абрахама при вышеуказанных температурах. Как видно из рис. 4 параметр Вендта-Абрахама монотонно уменьшается с увеличением концентрации меди, что характеризует увеличение степени локального структурного порядка в системе; значения уменьшаются при уменьшении температуры расплава. При этом параметр трансляционного порядка с увеличением концентрации меди изменяется незначительно, но зависит от температуры расплава (рис. 5): более высокое значение параметра при меньшей температуре.

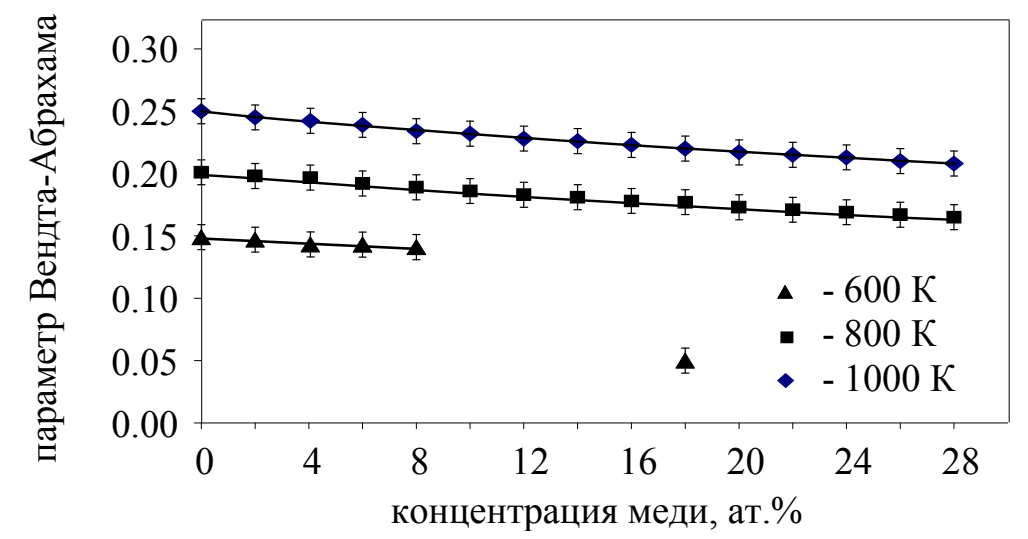

Рис. 4. Параметр Вендта-Абрахама в зависимости от концентрации меди в расплаве (аппроксимация полиномом 6-й степени)

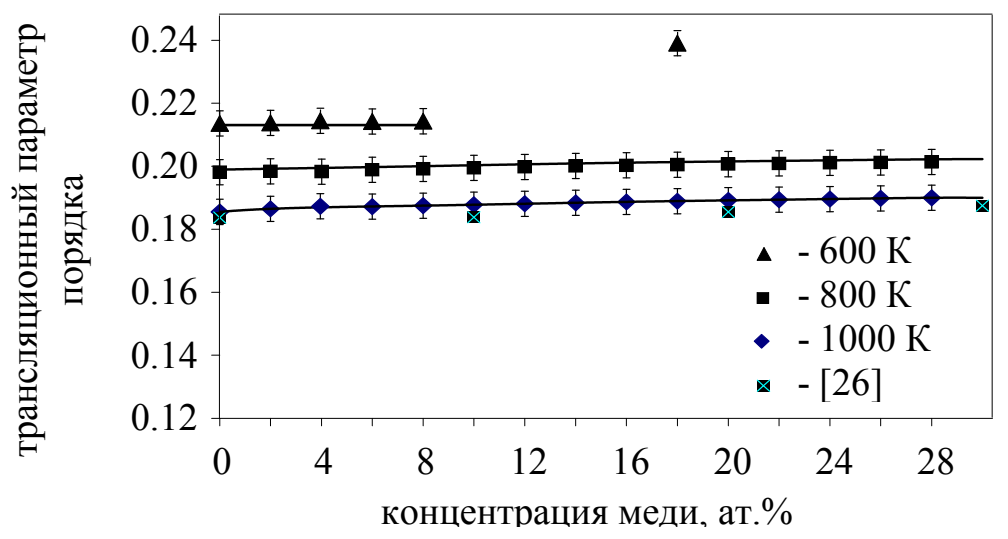

Рис. 5. Трансляционный параметр порядка в зависимости от концентрации меди в расплаве (аппроксимация полиномом 6-й степени)

Известно, что характер упорядочения атомов в расплавах влияет на физикохимические свойства расплавов, такие как вязкость, плотность, поверхностное натяжение и т.П., их температурно-концентрационные зависимости [14]. Мы рассчитали значения плотности расплавов при различных температурах в зависимости от концентрации меди в расплаве. На рис. 6, $a$ представлены результаты исследования. Как видно из рис. $6, a$ в области концентраций менее 17 ат.\% Сu отмечен линейный рост значений плотности расплавов. Представленные в настоящей работе расчетные значения плотности коррелируют с экспериментальными данными, представленными в работе [13], полученными высокоточным методом проникающего гамма-излучения [13] (рис. 6, б). В области концентраций 17 - 28 ат.\% Сu значения плотности также возрастают, но нелинейно (рис. 6), что согласуется с ранее полученными в [26] расчетами (рис.6, $a, \sigma$ ), выполненными с другими исходными параметрами. В [26] система $\mathrm{Al}_{100-\mathrm{x}} \mathrm{Cu}_{\mathrm{x}}$ получалась быстрым охлаждением равновесного расплава с температурой $\mathrm{T}=3000 \mathrm{~K}$ и состояла из 4000 атомов, расположенных в кубической ячейке с периодическими граничными условиями. Для приведения системы в состояние термодинамического равновесия было выполнено $4.5 \cdot 10^{6}$ временных шагов в NpT-ансамбле при внешнем давлении $\mathrm{p}=1.0$ бар. 

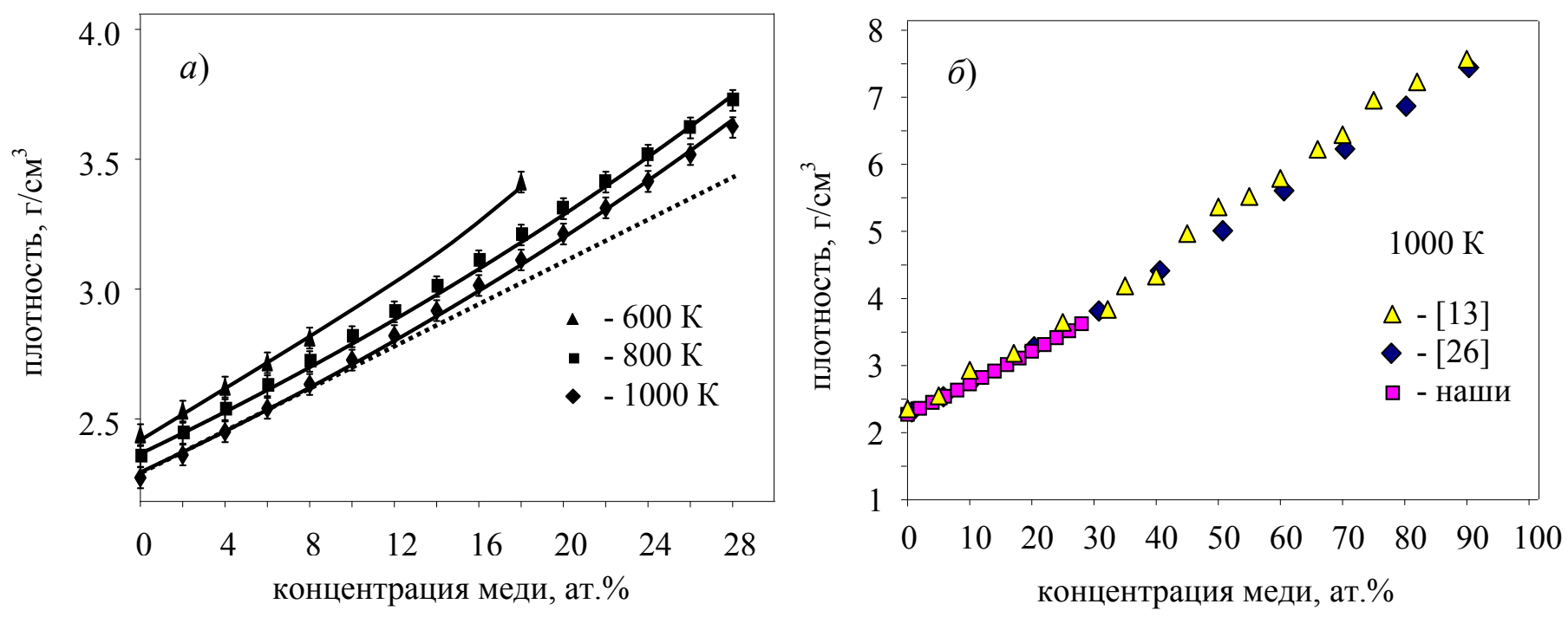

Рис. 6. Плотность расплавов Аl-Сu при различных температурах (a), в сравнении литературные данными при 1000 К (б)

\section{ЗАКЛЮЧЕНИЕ}

В работе в рамках численного эксперимента по моделированию молекулярной динамики расплавов решалась задача по исследованию локальных структурных особенностей ближнего порядка расплавов бинарной системы $\mathrm{Al}-\mathrm{Cu}$ в области, богатой алюминием. Данные моделирования были использованы для структурного анализа, выполненного с помощью параметра Вендта-Абрахама, функции радиального распределения $\mathrm{g}(\mathrm{r})$, трансляционного параметра порядка.

\section{ВЫВОДЫ}

1. В расплавах Al-Cu отмечено сильное взаимодействие между атомами разного сорта.

2. При $\mathrm{t}=600 \mathrm{~K}$ сильно переохлажденные расплавы некоторых исследованных составов стабильны, за исключением сплава состава близкого к эвтектическому, который при данных параметрах кристаллизуется.

3. Рост концентрации $\mathrm{Cu}$ в $\mathrm{Al}$ приводит к нелинейному росту значений плотности расплавов.

4. Результаты исследования согласуются с экспериментальными и расчетными литературными данными.

Полученные в настоящей работе расчетные данные по плотности могут быть использованы при анализе других структурно-чувствительных свойств исследованных расплавов, в частности, вязкости, в методе крутильных колебаний цилиндрического тигля с расплавом [27], а также в качестве справочного материала.

Сложный характер взаимодействия атомов в твердой фазе приводит к тому, что в жидкой фазе возникают микронеоднородности концентрационного и топологического (структурного) типов. Для уточнения характера ближнего атомного упорядочения в расплавах, типа упаковки атомов в этих микронеоднородностях и установления связи с твердым состоянием выполняются дополнительные комплексные исследования.

Работа выполнена в рамках государственного задания Министерства образования и науки РФ (Проект № 121030100001-3).

Компьютерное моделирование выполнено с использованием ресурсов кластера «Уран» на базе ИММ УрО РАН, г. Екатеринбург (https://parallel.uran.ru/).

Автор благодарит д.ф.-м.н. Рыльцева Р.Е. за помощь в выполнении работы. 


\section{СПИСОК ЛИТЕРАТУРЫ}

1. Зайцев А. И., Шимко Р. Ю., Арутюнян Н. А., термодинамических свойств и ассоциации в расплаве $\mathrm{Al}-\mathrm{Cu}$, их связи с условиями образования квазикристаллов // Доклады Академии наук. 2007. Т. 414, № 3. С. 352-356.

2. Семененко В. Е., Касилов А. А., Коваленко Т. А. Влияние термообработки на кинетику старения естественного микрокомпозита $\mathrm{Al}-\mathrm{CuAl}_{2} / /$ Вісник Харківського національного університету імені В.Н.Каразіна. Серія фізична "Ядро, частинки, поля". 2012. № 991, вып. 1(53). С. 90-94.

3. Mudry S., Shtablavyi I., Rybicki J. Correlation between the structure in the liquid state and the structure in the solid state in the $\mathrm{Al}-\mathrm{Al}_{2} \mathrm{Cu}$ eutectic alloy // Журнал фізичних досліджень. 2011. Т. 15, № 1, C. $1601-1-1601-5$.

4. Brillo J., Bytchkov A., Egry I., Hennet L., Mathiak G., Pozdnyakova I., Price D. L., Thiaudiere D., Zanghi D. Local structure in liquid binary Al-Cu and Al-Ni alloys // Journal of Non-Crystalline Solids, 2006, vol. 352, iss. 38-39, pp. 4008-4012.

5. Xiufang B., Xuemin P., Xubo Q., Minhua J. Medium-range order clusters in metal melts // Science in China Series E Technological Sciences, 2002, vol. 45, no. 2, pp. 113-119.

6. Меньшикова С. Г., Бельтюков А. Л., Ладьянов В. И. Об особенностях вязкости расплавов $\mathrm{Al}_{70} \mathrm{Cu}_{30}$ и $\mathrm{Al}_{65} \mathrm{Cu}_{35} / /$ Вестник Казанского технологического университета. 2014. Т. 17, № 23. C. $140-143$.

7. Mihua Sun, Xiufang Bian. Abnormal changes in the viscosity behavior of $\mathrm{Al}_{80} \mathrm{Cu}_{20}$ melt and its relationship to the glass-forming ability // Materials Letters, 2002, vol. 56, iss. 5, pp. 620-623.

8. Константинова Н. Ю., Курочкин А. Р., Борисенко А. В., Филиппов В. В., Попель П. С. Вязкость расплавов медь-алюминий // Расплавы. 2016. № 2. С. 157-164.

9. Plevachuk Y., Sklyarchuk V., Yakymovych A., Eckert S., Willers B., Eigenfeld K. Density, viscosity, and electrical conductivity of hypoeutectic Al-Cu liquid alloys // Metallurgical and Materials Transactions A, 2008, vol. 39, pp. 340-345.

10. Лыкасов Д. К., Чикова О. А. Вязкость жидких сплавов Al-Cu // Расплавы. 2007. № 4. C. 31-36.

11. Замятин В. М., Насыйров Я. А., Классен Н. И. и др. Аномалии на политермах вязкости жидких сплавов системы алюминий - медь // Журнал физической химии. 1986. Т. 60, № 1. С. 243-245.

12. Schick M., Brillo J., Egry I., and Hallstedt B. Viscosity of $\mathrm{Al}-\mathrm{Cu}$ liquid alloys: measurement and thermodynamic description // Journal of Materials Science, 2012, vol. 47, pp. 8145-8152.

13. Курочкин А. Р., Попель П. С., Ягодин Д. А., Борисенко А. В., Охапкин А. В. Плотность сплавов медь-алюминий при температурах до $1400{ }^{\circ} \mathrm{C}$ по результатам измерений гамма-методом // Теплофизика высоких температур. 2013. Т. 51, № 2. С. 224-232.

14. Бродова И. Г., Попель П. С., Барбин Н. М., Ватолин Н. А. Расплавы как основа формирования структуры и свойств алюминиевых сплавов. Екатеринбург: УрО РАН, 2005. 369 с.

15. Hultgren R., Desai P. D., Gleiser M., Hawkins D. T. Selected Values of the Thermodynamic Properties of Binary Alloys. Park, Ohio: American Society for Testing and Materials, 1973. 1442 p.

16. Dinsdale A. T., Quested P. N. The viscosity of aluminium and its alloys--A review of data and models // Journal of Materials Science, 2004, vol. 39, iss. 24, pp. 7221-7228.

17. Kawai Y. and Shiraishi Y. Handbook of Physico-Chemical Properties at High Temperature: The 140th Committee of Japan Society for Promotion of Science. Tokyo: ISIJ, 1988.

18. Хуснутдинов Р. М., Мокшин А. В. Локальный структурный порядок и одночастичная динамика в металлическом стекле // Известия РАН. Серия физическая. 2010. Т. 74. С. 677-680.

19. Замятин В. М., Баум Б. А. Неравновесность металлического расплава и другие факторы, определяющие качество металлопродукции // Расплавы. 2010. № 3. С. 12-20.

20. Xiufang Bian, Pan Xuemin, Qin Xubo, Jiang Minhua. Medium-range order clusters in metal melts // Science in China - Series E: Technological Sciences, 2002, vol. 45, no. 2, pp. 113-119.

21. Диаграммы состояния двойных металлических систем: Справочник. В 3-х томах. Том $1 /$ под ред. Н.П. Лякишева. М.: Машиностроение, 1996. 992 с.

22. March N. H., Tosi M. P. Atomic dynamics in liquids. New York: Dover, 1991 (first published 1976). $337 \mathrm{p}$.

23. Wendt H. R. Abraham F. F. Empirical criterion for the transition region based on Monte Carlo simulations // Physical Review Letters, 1978, vol. 41, iss. 18, pp. 1244-1260.

24. Tanaka H. Simple physical explanation of the unusual thermodynamic behavior of liquid water // Physical Review Letters, 1998, vol. 80, iss. 26, pp. 5750-5760. 
25. Cai J., Ye Y. Y. Simple analytical embedded-atom-potential model including a long-range force for fcc metals and their alloys // Physical Review B, 1996, vol. 54, iss. 12, pp. 8398-8410.

26. Хуснутдинов Р. М., Мокшин А. В., Меньшикова С. Г., Бельтюков А. Л., Ладьянов В. И. Вязкостные и акустические свойства расплавов $\mathrm{AlCu} / /$ Журнал экспериментальной и теоретической физики. 2016. Т. 149, № 5. С. 994-1004.

27. Бельтюков А. Л., Ладьянов В. И. Автоматизированная установка для определения кинематической вязкости металлических расплавов // Приборы и техника эксперимента. 2008. № 2. C. $155-161$.

\author{
Molecular Dynamic Simulation of the Local Structure of Al-Cu Melts in Liquid and Supercooled \\ States \\ ${ }^{1,2}$ Menshikova S. G. \\ ${ }^{1}$ Udmurt Federal Research Center, Ural Branch of the Russian Academy of Sciences, Izhevsk, Russia \\ ${ }^{2}$ Udmurt State University, Izhevsk, Russia
}

SUMMARY. Molecular dynamics calculations were performed using the LAMMPS program. The system under study consisted of 19986 atoms interacting through the EAM potential. Simulations were performed in an $\mathrm{NpT}$-ensemble at pressure of zero. We considered 15 different compositions of $\mathrm{Al}_{100-\mathrm{x}} \mathrm{Cu}_{\mathrm{x}}$ $(\mathrm{x}=0,2, \ldots, 28$ at.\%). The structural features of the melts were studied using the radial distribution function, the Wendt-Abraham parameter, and the translational order parameter. It is shown that strong interactions between atoms of different types are noted in Al-Cu melts. At a temperature of $600 \mathrm{~K}$, strongly supercooled melts of some of the investigated compositions are stable, with the exception of an alloy with a composition close to eutectic, which crystallizes at these parameters. An increase in the concentration of $\mathrm{Cu}$ in $\mathrm{Al}$ leads to a nonlinear increase in the values of the density of melts. The results of the study are consistent with the experimental and calculated literature data. The calculated density data obtained in this work can be used to analyze other structure-sensitive properties of the investigated melts, in particular, viscosity, in the method of torsional vibrations of a cylindrical crucible with a melt, and also as a reference material. The complex nature of the interaction of atoms in the solid phase leads to the appearance of microinhomogeneities of the concentration and topological (structural) types in the liquid phase. To clarify the nature of the short-range atomic ordering in melts, the type of atomic packing in these microinhomogeneities, and to establish a connection with the solid state, additional comprehensive studies are performing.

KEYWORDS: liquid alloy, short-range order, structure, concentration, Wendt-Abraham parameter, translational order parameter, supercooled melt.

\title{
REFERENCES
}

1. Zaitsev A. I., Shimko R. Yu., Arutyunyan N. A., Dunaev S. F. A study of thermodynamic properties and association in the Al-Cu melt and their relation to quasicrystal formation conditions. Doklady Physical Chemistry, 2007, vol. 414, no. 1, pp. 115-119. https://doi.org/10.1134/S0012501607050065

2. Semenenko V. E., Kasilov A. A., Kovalenko T. A. Vliyanie termoobrabotki na kinetiku stareniya estestvennogo mikrokompozita $\mathrm{Al}_{-}-\mathrm{CuAl}_{2}$ [Influence of Heat Treatment on Kinetics of Senescence of the Natural Microcomposite of $\mathrm{Al}_{-} \mathrm{CuAl}_{2}$. Visnik Kharkivs'kogo natsional'nogo universitetu imeni V.N.Karazina. Seriya fizichna "Yadro, chastinki, polya" [The Journal of Kharkiv National University, Physical series "Nucleus, Particles, Fields"], 2012, no. 991, iss. 1(53), pp. 90-94. https://periodicals.karazin.ua/eejp/article/view/13875/13061

3. Mudry S., Shtablavyi I., Rybicki J. Correlation between the structure in the liquid state and the structure in the solid state in the $\mathrm{Al}-\mathrm{Al}_{2} \mathrm{Cu}$ eutectic alloy. Journal of Physical Studies, 2011, vol. 15, no. 1, pp. 1601-1 - 1601-5. http://nbuv.gov.ua/UJRN/jphd_2011_15_1_12

4. Brillo J., Bytchkov A., Egry I., Hennet L., Mathiak G., Pozdnyakova I., Price D. L., Thiaudiere D., Zanghi D. Local structure in liquid binary Al-Cu and Al-Ni alloys. Journal of Non-Crystalline Solids, 2006, vol. 352, iss. 38-39, pp. 4008-4012. https://doi.org/10.1016/j.jnoncrysol.2006.08.011 
5. Xiufang B., Xuemin P., Xubo Q., Minhua J. Medium-range order clusters in metal melts. Science in China Series E Technological Sciences, 2002, vol.45, no. 2, pp. 113-119. http://dx.doi.org/10.1360/02ye9014

6. Men'shikova S. G., Bel'tyukov A. L., Lad'yanov V. I. Ob osobennostyakh vyazkosti rasplavov $\mathrm{Al}_{70} \mathrm{Cu}_{30} \mathrm{i} \mathrm{Al}_{65} \mathrm{Cu}_{35}$ [About special features of viscosity of melts of $\mathrm{Al}_{70} \mathrm{Cu}_{30}$ and $\mathrm{Al}_{65} \mathrm{Cu}_{35}$ ]. Vestnik Kazanskogo tekhnologicheskogo universiteta [Bulletin of the Technological University], 2014, vol. 17, no. 23, pp. 140-143.

7. Mihua Sun, Xiufang Bian. Abnormal changes in the viscosity behavior of $\mathrm{Al}_{80} \mathrm{Cu}_{20}$ melt and its relationship to the glass-forming ability. Materials Letters, 2002, vol. 56, iss. 5, pp.620-623. http://dx.doi.org/10.1016/S0167-577X(02)00565-7

8. Konstantinova N. Y., Popel' P. S., Kurochkin A. R., Borisenko A. V., Filippov V. V. Viscosity of aluminum-copper melts. Russian metallurgy (Metally), 2016, no. 2, pp. 144-149. https://doi.org/10.1134/S0036029516020075

9. Plevachuk Y., Sklyarchuk V., Yakymovych A., Eckert S., Willers B., Eigenfeld K. Density, viscosity, and electrical conductivity of hypoeutectic Al-Cu liquid alloys. Metallurgical and Materials Transactions A, 2008, vol. 39, pp. 340-345. http://dx.doi.org/10.1007/s11661-008-9659-2

10. Lykasov D. K., Chikova O. A. Vyazkost' zhidkikh splavov Al-Cu [Viscosity of al-cu liquid alloys]. Rasplavy [Melts ], 2007, no. 4, pp. 31-36.

11. Zamyatin V. M., Nasyyrov Ya. A., Klassen N. I. i dr. Anomalii na politermakh vyazkosti zhidkikh splavov sistemy alyuminiy - med' [Anomalies in the viscosity polytherms of liquid alloys of the aluminum - copper system]. Zhurnal fizicheskoy khimii [Journal of Physical Chemistry], 1986, vol. 60, no. 1, pp. 243-245.

12. Schick M., Brillo J., Egry I., and Hallstedt B. Viscosity of $\mathrm{Al}-\mathrm{Cu}$ liquid alloys: measurement and thermodynamic description. Journal of Materials Science, 2012, vol.47, pp.8145-8152. https://doi.org/10.1007/s10853-012-6710-x

13. Kurochkin A. R., Popel' P. S., Yagodin D. A., Borisenko A. V., Okhapkin A. V. Density of copper-aluminum alloys at temperatures up to $1400^{\circ} \mathrm{C}$ determined by the gamma-ray technique. High Temperature, 2013, vol. 51, pp. 197-205. https://doi.org/10.1134/S0018151X13020120

14. Brodova I. G., Popel' P. S., Barbin N. M., Vatolin N. A. Rasplavy kak osnova formirovaniya struktury i svoystv alyuminievykh splavov [Melts as the basis for the formation of the structure and properties of aluminum alloys]. Yekaterinburg: UrO RAN Publ., 2005. 369 p.

15. Hultgren R., Desai P. D., Gleiser M., Hawkins D. T. Selected Values of the Thermodynamic Properties of Binary Alloys. Park, Ohio: American Society for Testing and Materials, 1973. 1442 p.

16. Dinsdale A. T., Quested P. N. The viscosity of aluminium and its alloys--A review of data and models. Journal of Materials Science, 2004, vol.39, iss. 24, pp. 7221-7228. https://doi.org/10.1023/B:JMSC.0000048735.50256.96

17. Kawai Y. and Shiraishi Y. Handbook of Physico-Chemical Properties at High Temperature: The 140th Committee of Japan Society for Promotion of Science. Tokyo: ISIJ, 1988.

18. Khusnutdinoff R. M., Mokshin A. V. Local Structural Order and Single-Particle Dynamics in Metallic Glass. Bulletin of the Russian Academy of Sciences: Physics, 2010, vol. 74, no. 5. pp. 640-643. https://doi.org/10.3103/S1062873810050163

19. Zamyatin V. M., Baum B. A. Neravnovesnost' metallicheskogo rasplava i drugie faktory, opredelyayushchie kachestvo metalloproduktsii [Disbalans of metallic melt and other factors defined of quality of metalloproducts]. Rasplavy [Melts], 2010, no. 3, pp. 12-20.

20. Xiufang Bian, Pan Xuemin, Qin Xubo, Jiang Minhua. Medium-range order clusters in metal melts. Science in China - Series E: Technological Sciences, 2002, vol.45, no. 2, pp. 113-119. http://dx.doi.org/10.1360/02ye9014

21. Diagrammy sostoyaniya dvoynykh metallicheskikh sistem: Spravochnik. V 3-kh tomakh. Tom 1 [State Diagrams of Binary Metallic Systems: A Handbook. In 3 volumes. Volume 1]. Pod red. N.P. Lyakisheva. Moscow: Mashinostroenie Publ., 1996. 992 p.

22. March N. H., Tosi M. P. Atomic dynamics in liquids. New York: Dover, 1991 (first published 1976). 337 p. https://doi.org/10.1007/978-1-349-00929-9

23. Wendt H. R. Abraham F. F. Empirical criterion for the transition region based on Monte Carlo simulations. Physical Review Letters, 1978, vol.41, iss. 18, pp. 1244-1260. https://doi.org/10.1103/PhysRevLett.41.1244

24. Tanaka H. Simple physical explanation of the unusual thermodynamic behavior of liquid water. Physical Review Letters, 1998, vol. 80, iss. 26, pp. 5750-5760. https://doi.org/10.1103/PhysRevLett.80.5750 
25. Cai J., Ye Y. Y. Simple analytical embedded-atom-potential model including a long-range force for fcc metals and their alloys. Physical Review B, 1996, vol. 54, iss. 12, pp. 8398-8410. https://doi.org/10.1103/PhysRevB.54.8398

26. Khusnutdinoff R. M., Mokshin A. V., Menshikova S. G., Beltyukov A. L., Ladyanov V. I. Viscous and acoustic properties of AlCu melts // Journal of Experimental and Theoretical Physics, 2016, vol. 122, no. 5. pp. 859-868. https://doi.org/10.1134/s1063776116040166

27. Bel'tyukov A. L., Lad'yanov V. I. An automated setup for determining the kinematic viscosity of metal melts. Instruments and Experimental Techniques, 2008, vol. 51, no. 2, pp. 304-310. https://doi.org/10.1007/s10786-008-2027-z

Меньшикова Светлана Геннадьевна, кандидат физико-математических наук, старший научный сотрудник НЦ МФМ УдмФИЦ УрО РАН, доиент кафедры общей физики УдГУ,

mел.+7(3412)21-65-66, e-mail: svetlmensh@mail.ru 